\title{
Associative Relevance Based Eye Fixations Enhance Decision Making Processes in Scene Perception
}

\author{
Gufran Ahmad \\ College of Computer Sciences and Information Systems \\ Jazan University, Jazan45142, Saudi Arabia
}

Tel: 966-50-30-820-48Ｅ-mail: gufran.researcher@gmail.com

Received: Jan. 12, 2016

doi:10.5296/jmr.v8i2.8852
Accepted: Feb. 16, $2015 \quad$ Published: April 1, 2016

URL: http://dx.doi.org/10.5296/jmr.v8i2.8852

\begin{abstract}
Recent research studies on eye movements in area of information processing task, such as scene perception have magnificently advanced towards understandings of underlying visual perception mechanism and human cognitive dynamics. Besides, business applications of eye tracking are unceasingly revealing innovative trends based on pragmatic scenarios. In this study, we conducted a number of eye tracking experiments to establish our hypothesis that the eye fixations based on the associative relevance found within the contexts of scenes during scene perception significantly bettered the processes of decision-making. The collected eye movement data from participants who viewed artistic scenes discovered that the tracks of eye fixations traversed along the existing associative relevance among the elements of scenes for decision-making processes. These experimental evidences confirmed our hypothesis that the associative relevance based eye fixations enhanced decision-making processes in scene perception.
\end{abstract}

Keywords: Decision making, Eye fixations, Associative relevance, Scene perception 


\section{Introduction}

\subsection{Introduction and Importance}

Studies on eye movements are undergoing in the advanced phase of innovative approaches and solutions. A number of interdisciplinary demands and interests have extended eye movement researches into numerous perspectives of social, economic, business, and scientific scenarios. Research areas like, human and developmental psychology, psycholinguistic and readings, neuroscience, vision research, usability studies, business marketing and advertising research, ophthalmology, human computer interaction are trending for more profound interests than ever before and eye movement studies are taking part in these areas. Additionally, these studies have improved research visions and insights, tools and techniques, and extensive applications. Tracking of eye movements leads to noteworthy comprehensions of human mind, in terms of human intentions that are the strategic concerns for businesses because businesses can make acquainted with human conducts, and attitudes to make business plans and policies accordingly. Further, customers' opinions and purposes are necessities for better business and market behavior that mainly depends on their behaviors. Currently, a number of online and offline businesses, like marketing, advertisement, shopping, search engine optimization, and web designing are converting and revolutionizing their business strategies and objectives in accordance with the innovative outcomes of eye movements for business growths (Granka, Joachims, Gay, 2004; Bojko, 2013; Koller, Salzberger, Brenner, Walla, 2012; Pan, Hembrooke, Gay, Granka, Feusner, Newman, 2004; Hermansen, 2015; Pan, Hembrooke, Joachims, Lorigo, Gay, Granka, 2007; Ahmad, 2015).

Usually, eye movements involve in the verbal and nonverbal communications and assist in providing the desired information among the participants of communication. In addition to these, eye movements actively contribute in the processing of data for information and the visualization of information that are common practices among professionals and individuals, including artists and scientists. In reality, this complex mechanism of neurocognition is a combination of numerous underlying processes existing in human mind. The human mind incites and causes cognitive processes, like human's sensation, consciousness, visual attention, perception, meta-cognition, reasoning, analogical thoughts, information processing, and other concerned processes (Wells, 2000; Tsotsos, 2011; Tommasi, Peterson, Nadal, 2009; Stark, Ellis, 1981; Snowden, Thompson, Troscianko, 2012; Holsanova, 2008; Rayner, 1992; Bly, 1999; Ahmad, 2011; Ahmad, 2013; Ahmad; 2014; Ahmad, 2015).

In eye movement processes, eyes change their gazes to spot a specific portion of the visible region in viewing because of having tendency to perceive the degree of detail visible in the central direction of gaze. In the movements, they pass through two temporal phases: fixations (the stops or periods of time when point of gaze or significant look is relatively slow) and saccades (the hops between stopping points). Saccades are often information seeking and directed to specific objects or regions by the requirements of ongoing behavior. This infers the existence of cognitive processes of eye movements in viewing as well. Therefore, the underlying mechanism of visual viewing is sequential and coordinated phenomena of cognitive as well as correlated processes (Bojko, 2013; Brown, 1999; Duchowski, 2003; 
Gompel, Fischer, Murray, Hill, 2007; Henderson, Hollingworth, 1998; Henderson, Hollingworth, 1999; Hoffman, 1998; Holsanova, 2008; Liversedge, Gilchrist, Everling, 2011; Rayner, 1992; Snowden, Thompson, Troscianko, 2012; Stark, Ellis, 1981; Ahmad, 2014; Ahmad, 2015).

\subsection{Related Background and Corresponding Research Design}

The study of relationships of fixation sequences of eye movements to the behavior of usual human activities has its origin. In fact, inherent salience of objects is not accountable for focal shifts in eye movements, but by their importance to the task or context in hand. In contradiction of free viewing, the movements of eyes and contextual actions are associative in nature and have a chain of linking. Moreover, contextual actions compose of a number of perspectives including an act of associativity within the contexts, elements, or intents. Such associativity has built upon the sense of relevance among the contexts of the object (Stark, Ellis, 1981; Snowden, Thompson, Troscianko, 2012; Rayner, 1992; Nikolaev, Pannasch, Ito, Belopolsky, 2014; Liversedge, Gilchrist, Everling, 2011; Holsanova, 2008; Hoffman, 1998; Henderson, Hollingworth, 1999; Henderson, Hollingworth, 1998; Griffin, 2004; Gompel, Fischer, Murray, Hill, 2007; Ahmad, 2015).

In addition, a focused visual appearance, i.e. spotlight metaphor, enables and constructs the visual focus of attention in eye movements during artistic scene viewing. The center of focused attention in visual area is considered as having more brightness than areas to which attention is not focused or areas from which attention has been detached. The spotlight of attention switches off at one location and then switches on at other. Besides, as human's capabilities for attention and processing for information has restraints cognitively, so it is rather challenging to consider about focusing everything at a time. Cognitively, human gives attention to small part of visual object, one at a time and likewise, human can focus on only small piece of information at a time. This is why the human processing of information is reasonably gradual as it works on the mechanism of human cognition (Bly, Rumelhart, 1999; Gompel, Fischer, Murray, Hill, 2007; Griffin, 2004; Hoffman, 1998; Holsanova, 2008; Liversedge, Gilchrist, Everling, 2011; Nikolaev, Pannasch, Ito, Belopolsky, 2014; Snowden, Thompson, Troscianko, 2012; Stark, Ellis, 1981).

As a further matter, there occur numerous impact factors, which engender reasoning, notion of analogy, flow of thoughts, meta-cognition, and other pertinent cognitive processes during the eye movements. We regard in a location of scene that is partly determined by the scene's constraints and region's informative description, partly by the task, intent, context, or interest. Viewers can arrange diverse visual paths through the same scene, since they extract information from those parts of the scene to describe particularly. Therefore, the evolved flow of thoughts cognitively, motivates the conscious focus of attention to change to the next contextual part of the scene. By doing so, these impacting factors propel human visual focus of attention dynamically in the phenomena of eye movements (Wells, 2000; Tsotsos, 2011; Stark, Ellis, 1981; Rayner, 1992; Liversedge, Gilchrist, Everling, 2011; Holsanova, 2008; Hoffman, 1998; Henderson, Hollingworth, 1999). 
Normally, an art is a human way of cognitive activities and the art has the purpose to influence the minds of people who looked at them. Art is productive activity that focuses on the thoughtful modification and embellishment of worldviews. Mostly, all known pieces of art are creative and metacognitive as per their roles because they are self-explanatory. The types and styles of art are technology-driven as innovative technologies bring renaissance to the artworks. The most essential part of art is its motive to become conscious about itself and in turn, firing up the cognitive processes in human mind. In addition, as science is laying its foundation to understand our knowledge about art, likewise, the art offers us a view of mind that understands the art. Undoubtedly, we identify that all types of art are one of the fabulous representations in our lifetimes. It can calm down our distressed heart and motivate our mental states and spirits. Further, artistic perceptions stimulate profound thoughts as well as all types of sensations. Along with other sensations and perceptions, an art stimulates the human emotions as well. That is an integral part of human intelligence, so that the onlookers of artworks may sense and perceive a novel lookout (Holsanova, 2008; Solso, 1994; Solso, 2003; Turner, 2006; Viegas, Wattenberg, 2007; Ahmad, 2011; Ahmad, 2013; Ahmad, 2014; Ahmad, 2015).

During artistic scene observation, we move our eyes rapidly in irregular manner to change focus from one fixation to another fixation. This process, saccade, is one of the most common behavior of eyes. Pattern is obtained only during the periods of relative gaze constancy, known as fixations. The process of directing the eyes to view picture in real time is known as gazing of eyes. The processes of eye movements bring about and propagate a series of streaming thoughts successively to obtain information about the scene or object of interest. These streams of thoughts predominantly originate the notion of analogy. Although there are a number of standpoints about analogy itself, yet analogical impression is constantly accessible in visual perceptions. Based on existing research works, it gives the consideration that the standpoint of mapping and the standpoint of higher level of perception are two dissimilar looks of the same thing, i.e., analogy. Analogy is one of the inherent concepts that persist during the phenomena of eye movements, although it is not the complete reason to believe as this does not explain the whole scenario (Wells, 2000; Viegas, Wattenberg, 2007; Turner, 2006; Tsotsos, 2011; Solso, 2003; Solso, 1994; Holsanova, 2008; Hofstadter, Sander, 2013; Gentner, Rattermann, Forbus, 1993; Gentner, Medina, 1998; Gentner, Markman, 1997; Gentner, 1983).

There comes the process of associative relevance that are progressive and coexisting cognitive processesand are emanated from thoughts of analogy and continue to flow during entire timespan of visual scene viewing. These generated processes of associative relevance bring about the process of associations among the relevant entities or contexts. These associative relevance phenomena pass through human cognitive process as an aid during scene viewing, take part in focal shifts of eyes during active scene viewing as well as facilitate in decision making process by choosing the optimalroute of alternative.Associative relevance is developmental and interrelated notion, which originates from thoughts of analogy during the process of human viewing. The influence of associative relevance predominantly remains during cognitive phenomena of eye movements. It is an underlying 
mechanism for creativity and annihilation of complexity during information processing and information retrieval for better interpretation of the object of interests. Besides, it resembles as an associative chain that links contexts, intents, portions, elements, shapes, sizes, colors, contrasts, or relations based on similarity or sameness. Further, associative relevance manipulates and generates an incitement cognitively to associate relative contexts, intents, elements, etc. The process of associative relevance initiates during the shifts in visual focus of attention as the movements of eyes establish associations among analogical contexts or portions of interest during the active scene viewing (Gentner, 1983; Gentner, Markman, 1997; Gentner, Medina, 1998; Gentner, Rattermann, Forbus, 1993; Gompel, Fischer, Murray, Hill, 2007; Holsanova, 2008; Ahmad, 2014; Ahmad, 2015).

\subsection{Decision Making Process and Proposed Hypothesis}

Sited literatures (Stark, Ellis, 1981; Rayner, 1992; Liversedge, Gilchrist, Everling, 2011; Holsanova, 2008; Hoffman, 1998; Henderson, Hollingworth, 1998; Griffin, 2004; Gompel, Fischer, Murray, Hill, 2007) suggest that human eye saccades are information seeking in nature and are engaged in the job of retrieving information. However, the fixations play significant role, as they get involved in decision making, so that new information based on the previous decision might be sought. Further, the decision might be verified for future purposes as well. Besides, the eye fixations act as junctions that undergo through the processes of decision-making under the associated cognitive processes of influential nature (Adair, 2010; Bird, Lauwereyns, Crawford, 2012; Nikolaev, Pannasch, Ito, Belopolsky, 2014; Orquin, Loose, 2013; VanRullen, Thorpe, 2001).

The movements of eyes consisting of fixations and saccades have a clearly defined objective that may be organized in several alternative ways of movements by making a decision based upon choosing from a set of possible alternatives under the guidance of the cognitive processes and analogical thoughts. Each choice offers its own advantages and disadvantages, so that in a complex situation, the decision maker might not be able to make a preferable option at once and quickly decide why he or she should prefer one alternative and not another. To clarify the situation and compare the alternatives in several aspects, inherent human information processing along with associative relevance phenomena suggest a series of cognitive operations. Their aims are to analyze the situation critically and thus prepare a decision to move ahead during active scene viewing phenomena (Adair, 2010; Bird, Lauwereyns, Crawford, 2012; Holsanova, 2008; Nikolaev, Pannasch, Ito, Belopolsky, 2014; Orquin, Loose, 2013; VanRullen, Thorpe, 2001).

We propose the hypothesis that associative relevance based human eye fixations enhance decision making processes during the scene perception. This proposed hypothesis is completely based on cognitively generated underlying mechanism consisting of indivisible flow of thoughts that produces the process of associative relevance profoundly.

An undertaking (a system of actions initiated by eye fixations) having a clearly defined objective may be organized in several alternative ways (eye gazes) of fixations by making a decision based upon choosing from a set of possible alternatives produced by series of fixations. Each choice offers its own advantages and disadvantages, so that in a complex 
situation, the decision maker might not be able to make a preferable option at once and quickly decide why human should prefer one alternative and not another. To clarify the situation and compare the alternatives in several aspects, eye movements suggest a series of operations based on cognitively evolved associative relevance process. Their aim is to analyze the situation critically and thus prepare a decision for those bearing the responsibility for a final choice by decisive path of fixations based on associative relevance that ultimately assists in making decision within the contexts of visual scenario.

\section{Eye Tracking System}

In eye tracking system, the system illuminates infrared light for tracking the eye movements. The camera, connected to the system, captures the location of viewer's eyes in terms of fixation during experimentation time. As the viewer moves his/her eyes to look a new location of the scene, the camera records new fixation also. This process of recording continues subsequently. The system generates eye movement tracks and heat maps using the captured data which is utilized for further analysis.

The schematic diagram of eye tracking system and basic processes involved during eye tracking experimentation is represented in Figure 1.

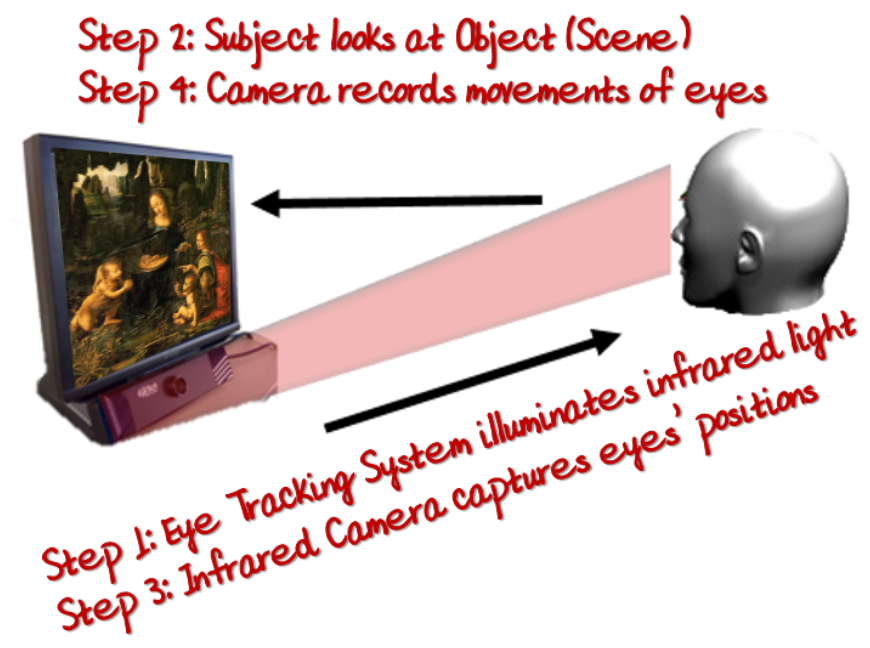

Figure 1. Eye tracking system with operational processes

The traces of eye movements are taken in diverse layouts as per analyst's suitability. Among them, there are two most common formats are Heat Map and Sequenced Gazing with circle of concentration. In Heat Map, the track of eye is recorded as illumination and intensity of infrared light rays. This is based on Energy Therapy Technique (ETT). In Sequenced Gazing, the eye tracks are entered as numbered circles with their areas indicating the time duration of eye's gazing in those areas respectively (Bojko, 2013; Brown, 1999; Duchowski, 2003; Holsanova, 2008).

In our experiments, we study track of eye fixations as the sequenced gazing of viewer's eye movements, which is generated by the system, during scene viewing. These are the dynamic shifts of eye gaze in scene viewing. 


\section{Present Study}

We investigate the gazing of eye movements from cognitive perspective, including the associative relevance process, during scene viewing and analyze the patterns of sequenced gazing to visualize the information. Here, visualization of sequenced gazing patterns to extract information for interpretation are essential steps of this study.

Initially, eye movements, in terms of sequenced gaze, are collected from participants who view full-color scenes while engaging in a visual search task in which they are freely viewing different fields of each scene. Finally, we compare and analyze the sequenced gazing against the artistic scene. The interpretation is carried out with the help of cognitive and analogical processes in current research.

The study on eye movements during scene viewing consists of a number of steps to be performed. These steps are represented as shown in the adjacent flow chart diagram (figure 2). This is a comparative study of two items; one item is artistic scene and other item is the eye movement tracks of the same scene, which is generated from eye tracking system.

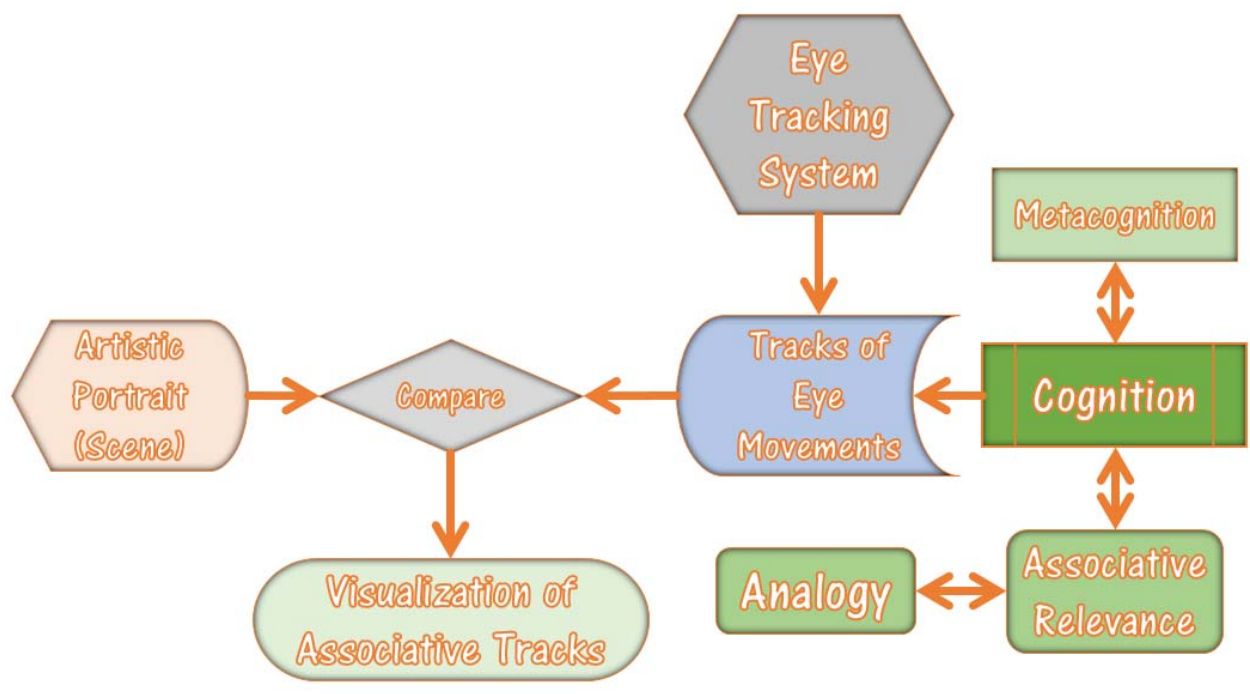

Figure 2. Flow chart of research study

It begins with recording of eye movement tracks for a Subject; a viewer on eye tracking system for an Object; artistic scene. The generated eye movement tracks of the same artistic scene is comparable to the original artistic scene. This comparative analysis infers visualization and interpretation of the outcome. So, we compare these two items side by side.

During comparison stage, we make use of cognitive process including metacognitive process, in addition to analogy based associative relevance mechanism that is happening consistently. These underlying processes generate crucial correlation that creates resultant maps. By analyzing, we come up with concluding remarks about the dependency of eye fixations on the associative relevance that controls and enhances the process of decision making in human mind. 


\section{Macrothink}

Journal of Management Research

ISSN 1941-899X 2016, Vol. 8, No. 2

\section{Method}

We selected 82 participants from a number of fields randomly, aging from 19 years to 54 years. Further, we assigned these participants (Subjects) to view three randomly selected famous artistic scenes (Objects) as shown below in figure 3.

The artistic scenes were "Virgin of the rocks (the Madonna of the rocks)", "the Virgin and child with saint Anne", "the holy infants embracing".
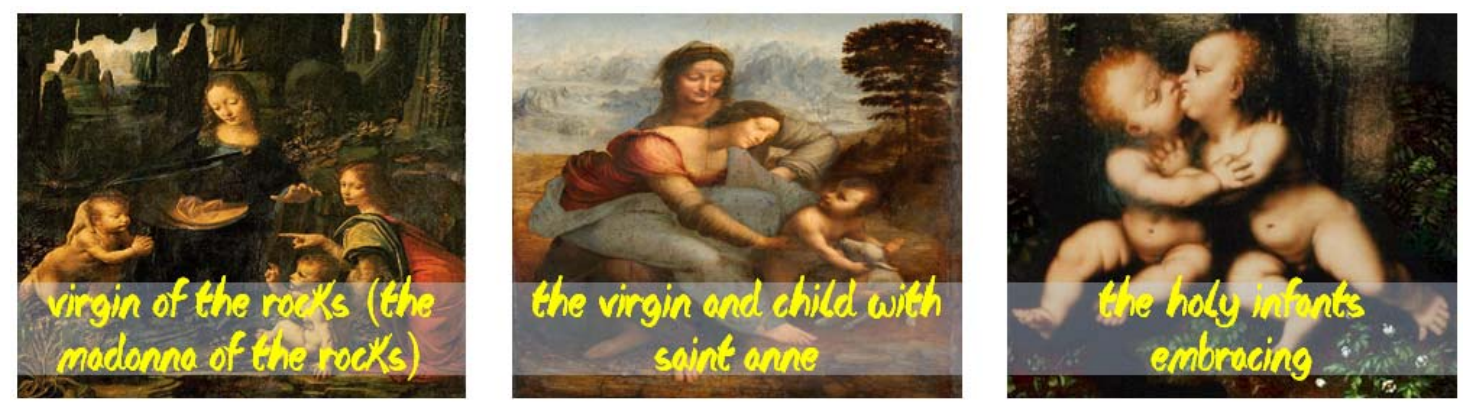

Figure 3. Selected Artistic Scenes for research study

Their eye movements were closely monitored as they viewed 32 bits full-color artistic scenes. The Objects, the scenes were displayed on a computer monitor. The scenes were shown at a resolution of $1280 \times 1024$ pixels and subtended $15 \mathrm{deg}$. horizontally by $10 \mathrm{deg}$. vertically at a viewing distance of $75 \mathrm{~cm}$. Eye position was sampled from an Eye Tech Digital Systems TM3 $16 \mathrm{~mm}$ Eye Tracker, and eye tracking data was parsed into sequenced gazing with circles of concentration.

The Subjects' heads were held steady in advance prior to experimentation. Prior to the first trial, Subjects completed a procedure to calibrate the output of the eye tracker against spatial positions on the display screen. This procedure was repeated regularly throughout the experiment to maintain high level of accuracy. Subjects were initiated to view the scenes freely.

The scenes were presented to the Subjects for maximum duration of 60 seconds. During this time span, the Subjects viewed the scenes with their normal eyes and focused attention on the Object, the scene.

Here, we analyzed all 3 scenes respectively with the intention to elaborate our findings in the most common and generalized perspective. 


\section{Analysis}

\subsection{Analysis 1: Study of "Virgin of the rocks (the Madonna of the rocks)" artistic scene}
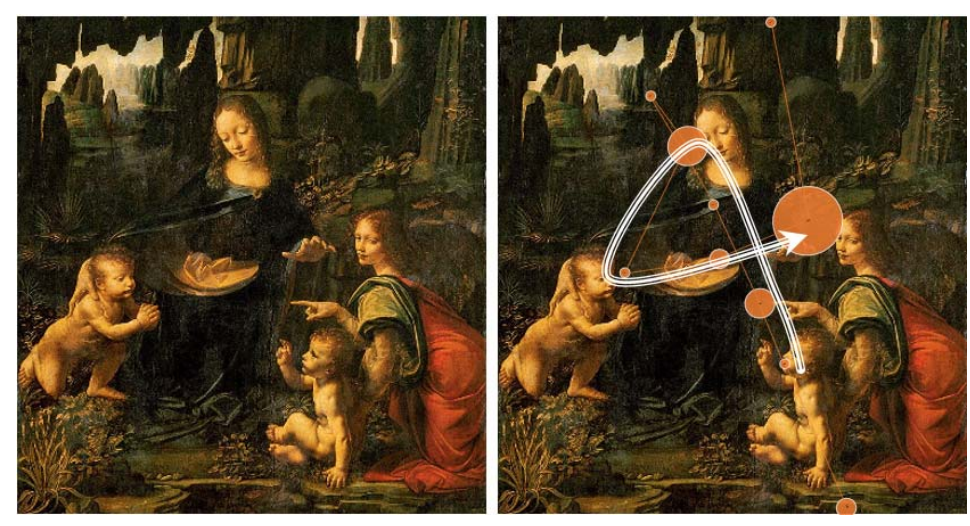

Figure 4. Virgin of rocks scene and eye movement track of the same artistic scene

In experimentations of the above artistic scene (figure 4), the left side scene is the original scene of Virgin of the rocks (Madonna of the rocks) showing Madonna and child Jesus along with the infant John the Baptist and an angel. The right side scene is the track of eye fixations by eye tracking system during the scene viewing by the Subject.

In beginning of the experimentation, as the Subjects started to move the eyes randomly, they looked at the lower right side of the scene. Subsequently, as the visual attentions and consciousness of the Subjects were gained due to happening of cognitive processes, they started to look at the infant John the Baptist and focused over the hand gestures and facial expression of the infant John the Baptist. At the moment, eye fixations were in need for a decisive move forward. This decision making process was carried along with inherently intrinsic processes of cognitively evolved associative relevance which was based on analogical thoughts that are flowing within cognitive minds.

Subsequently, by the coexisting phenomena of associative relevance evolved from analogical thinking, they shifted their eye fixations towards the facial expression of Virgin of the rocks (Madonna); just top central portion of the scene. At this state, the Subjects attained the process of chaining the relevant contexts of the scene in accordance with the existing process of associative relevance and perceived the inherent process that consistently directed and controlled the track of eye fixations. Further in the next step, the process of associative relevance in the Subjects' minds caused them to move along with their eye fixations across the facial expression and hand gesture of child Jesus. The procedure continued till the track of eye fixations reached to the central right-side of the scene where the hand gesture of the Virgin of rocks (Madonna) and facial expression of the Angel remained.

Therefore, we concluded that the process of associative relevance guided the eye fixations so that the eye fixations moved forward in decisive manner, in other words, a better decision is made by eye fixations under the influence of underlying mechanism of cognitively evolved process of associative relevance. 


\section{Ml Macrothink}

Further, later interview of Subjects confirmed the influential factor of associative relevance that led them to move their eyes decisively. In the beginning, they were gazing at the infant John the Baptist, later got interest in analogous contexts of gestures and facial expressions within the elements of scene. These factors helped and guided in making decision for the next move within various portions of the scene. The process of such viewing continued further and ended after allocated times pan. So, we concluded that in these experimentations, associative relevance based eye fixations enhanced the process of decision making in scene perception.

\subsection{Analysis 2: Study of "the Virgin and child with the Saint Anne" artistic scene}
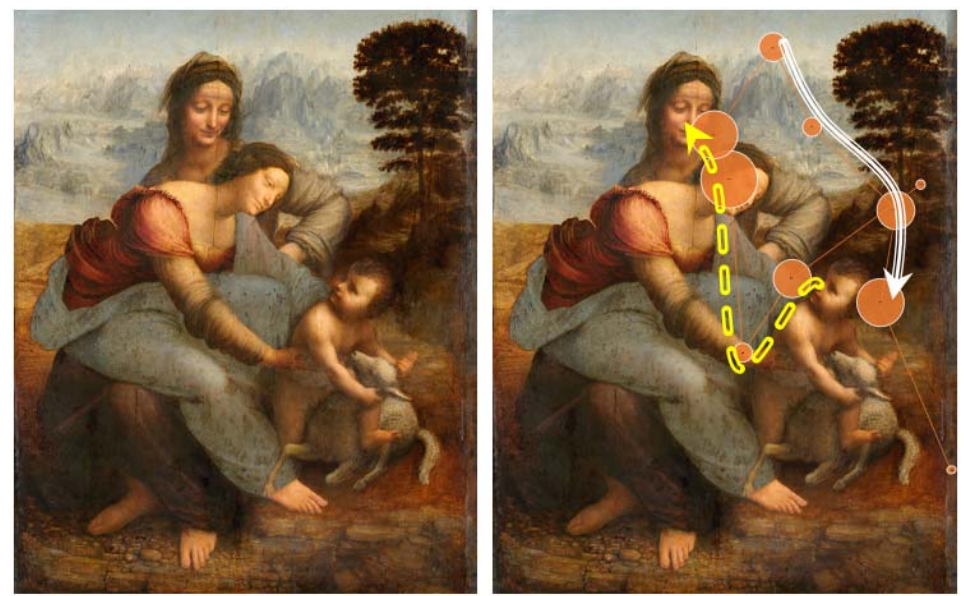

Figure 5. The Virgin and child with the saint Anne scene and eye movement track of the same scene

In this experimentations of artistic scene (figure 5), the left side scene is a famous painting named as the Virgin and Child with saint Anne that depicts saint Anne, her daughter the Virgin Mary and the infant Jesus. Christ is shown grappling with a sacrificial lamb symbolizing his passion as the Virgin tries to restrain him.

In the beginning of experimentations, the Subjects looked at the right-side region of the scene. Then, they looked at facial expression of the child Jesus in the scene due to attainment of the visual perception. This visual attention was built up on by undergoing cognitive processes that evolved flow of analogical thoughts. Ultimately, these flow of analogical thoughts sparked cognitively generated process of associative relevance. Based on these processes of associative relevance which were established on the basis of human emotion, the eye fixations decisively moved towards the hand gesture of the Virgin Mary. Subsequently, the track of eye fixations moved to the facial expression of the Virgin Mary and thereafter, the facial expression of the saint Anne. This brought the track of eye fixations towards upper side of the scene.

Later, due to the origination of a novel flow of thought and the breaking of the contextual factor of human emotion, associative relevance process could not further direct eye fixations on the same basis of human emotion rather the process emerged with a novel contextual factor of background sight of nature in terms of mountains. Subsequently, due to the dominating and coexisting phenomena of analogy, the flow of analogical thinking emanated 


\section{Ml Macrothink}

associative relevance that assisted eye fixations in making decision to move forward from a mountain to the next mountain. In the next subsequent moves, the eye fixations decisively followed along the guidance of associative relevance process based on contextual background sight of the nature consisting of mountains and contextual color oriented trees. However, these happenings of phenomenal sequences concluded that the associative relevance processes directed and guided the Subjects' eye fixations for making better decisions which is evident form the visualization of associative tracks of eye movements. Further, these observations led towards another remark that the track of eye fixations were totally controlled by the prominent factor, i.e. the process of associative relevance during this scene viewing.

In successive interview, the concerned Subjects revealed that they were strongly influenced by the human emotions and excited to know about the interrelated scenario within the scene. Mostly, they were interested to understand the human emotions and the background of the scene. This made them to shift their eyes as directed meaningfully by existing influential portions within the scene. Therefore, we concluded that the associative relevance based eye fixations enhanced the decision making process in scene perception.

\subsection{Analysis 3: Study of “The holy Infants embracing” artistic scene}

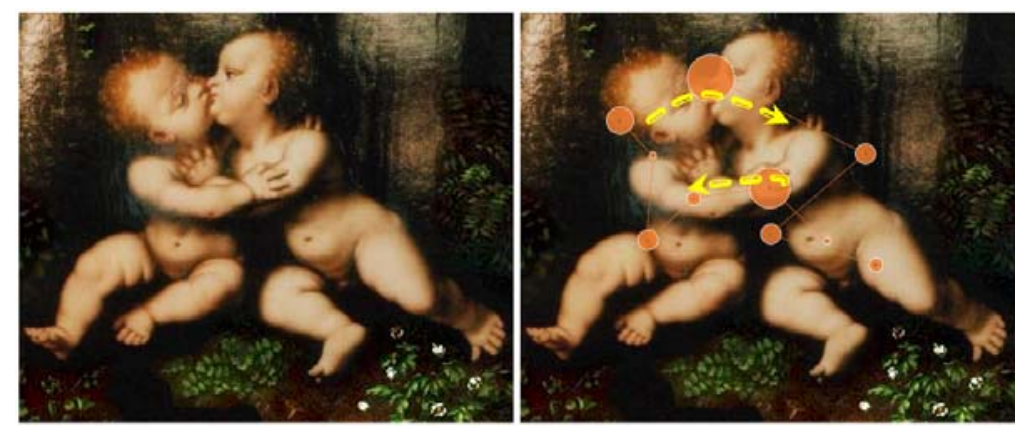

Figure 6. Scene of holy Infants embracing and eye movement track of the same scene

In these eye tracking experiments for the holy Infants embracing scene (figure 6), the scenery represents the infant Christ embracing his cousin John the Baptist. The scene at the left side is the original artistic scene, whereas the scene at the right side is the eye movement track of the same scene.

In the beginning, due to the initiation of visual attention and consciousness by viewing artistic scene, the Subjects focused eye fixations at the center portion of scene. It was due to difference in color contrast of context that compelled the focus of eye gazes to perceive the underlying cognizance. At this stage, the formation of cognitively evolved associative relevance among contextual human emotions (represented by hand gestures) by the flow of analogical thoughts played a significant role and made to move the eye fixations decisively. Later, the eye fixations shifted to the other portions of the scene. After that move, the eye fixations shifted randomly for a while for information seeking mode as it could not find associative relevance based context within the elements of scene.

Next, as the eye fixations came across the face of left infant of the scene, the visual attention and sensation of the Subjects perceived cognitively evolved associative relevance based on 
contextual human emotions. The existing facial expression of left infant had associative relevance with the facial expression of the right side infant. Hence, the eye fixations based on associative relevance made decision to move along facial expressions of the infants.

Hence, these experimentations showed the existence of cognitively created associative relevance processes and directed shifts in accordance with these associative relevance processes during the scene viewing.

Later interviews of the Subjects concluded that they got interest in shiny facial expressions of Infants as well as hand gestures of the Infants. These portions of the scene were attractive to them as these were full of human emotions. These related influencing factors were very peculiar to them. So, they decided to move their eyes accordingly.

So, these sequential activities led towards the observable confirmation that the existence of associative relevance process that streamed in between the human cognitive processes during scene viewing, guided and controlled the track of eye fixations. Therefore, we established that the associative relevance based eye fixations boosted decision making processes in scene perception.

\section{Discussion}

In this research work, we choose some of the finest pieces of art intentionally. The artistic sceneries embrace in their manifestation with the foremost purpose of artistic artworks in the shape of human cognition mechanism in viewing these artistic sceneries. These creative pieces of art reflect inherent human interaction to perceive knowledge and interpretation of realistic world in human mind. These emotional views are rather too complicated to understand from visual analytics and analytical reasoning. Consequently, these cognitive perspectives, i.e. thinking of analogy and process of associative relevance are discovered by the tracks of eye fixations in scene viewing (Wells, 2000; Viegas, Wattenberg, 2007; Turner, 2006; Solso, 2003; Solso, 1994; Liversedge, Gilchrist, Everling, 2011; Holsanova, 2008; Hofstadter, Sander, 2013).

Decision making has become a science rather than an art. Although in some of the management sectors, people still consider it as an art, yet the studies of decision making processes have revealed it as a science. In a number of cited literatures (Adair, 2010; Bird, Lauwereyns, Crawford, 2012; Holsanova, 2008; Nikolaev, Pannasch, Ito, Belopolsky, 2014; Orquin, Loose, 2013; VanRullen, Thorpe, 2001), the decision-making process is closely connected with the human cognition as there is a significant amount of contribution and control carried out by the human interaction. Moreover, most of the times, the decisions are made by the human within human-centric processes and scenarios.

Coexisting associative relevance processes from the flow of analogical thoughts during scene viewing is significant step for appropriate retrieval of task-relevant visual information which are essential for visualization of final maps. In this study, we observe that the generated eye movement tracks of fixations are strongly following the same path as directed and decided by the associative relevance processes. These facts show a clear tendency for decision making process to get initiation from the associative relevance based eye fixations. Therefore, the eye 
movements get manipulated operation under the guidance of associative relevance broadcast. As a result, without taking into account the influential factor; associative relevance propagation, it is impossible to link the entire scenario of human cognition in the sequential eye fixations of eye movement tracks.

The visualization of streaming associative relevance process in terms of followed eye movement tracks is a tactical and decisive part of whole activities. The visualization of decisive and controlled eye movement tracks, in terms of associative relevance, is unarguably innovative perspective of each and every analyst who examine them for definite intents of decision making processes. This, in turn, causes a number of associative relevance scenarios in scene viewing by various perspectives of analysts.

The directional shifts in eye fixations have correlated consequences along with the decision making processes that is happening cognitively within human mind and is consistently processing information together with the associative relevance processes. The visualization of relevant eye movement tracks reinforces again the existence of dominating associative relevance factor; the controller of entire phenomena in between the inherent cognitive and metacognitive processes during scene viewing.

In addition to these arguments, the experimental evidence of associative traversal path generated by eye tracks as the happening of decision making processes during active scene viewing holds our hypothesis for which we conducted a series of experimentations. The hypothesis that the associative relevance based eye fixations enhance decision making processes in scene perception, is persuasive and pioneering breakthrough related to eye movements study.

\section{Acknowledgement}

At first, our sincere and earnest thanks go to the participants who donated their precious time and efforts during the entire experimentations. We are indebted to their active participations in this research findings. Secondly, we are grateful to the financial supporter; Global IT Consultancy for Innovations without whom the current research work could not be carried out. Finally, we pay my special tribute to the great artist; Leonardo De Vinci for his splendid pieces of art.

\section{References}

Adair, J. (2010). Decision Making and Problem Solving Strategies. London, KoganPage Press.

Ahmad, G. (2015). Dynamic Shifts in Visual Focus of Eye Movements Emphasize Associative Relevancy. Research in World Economy, 6(4), 18-28. http://dx.doi.org/10.5430/rwe.v6n4p18

Ahmad, G. (2015). Visual Focus of Attention Actively Associates Relevancy in Eye Movements. Journal of Business Theory and Practice, 3(2), 209-223. 
Ahmad, G. (2015). Associative Relevance Based Stimulus Shifts Focus in Eye Movements, International Business Research, 8(10), 25-34. http://dx.doi.org/10.5539/ibr.v8n10p25

Ahmad, G. (2015). Eye Fixation curves along Analogical Thinking in Scene Viewing. International Journal of Engineering and Industries, 6(1), 54-62.

Ahmad, G. (2014). Flow of Analogical Thoughts Controls Eye Movements in Scene Viewing, Journal of Next Generation Information Technology, 5(4), 118-125.

Ahmad, G. (2014). Dynamics of Eye Gazing rely on Associative Relevance in Scene Viewing. Journal of Convergence Information Technology, 9(2), 35-42.

Ahmad, G. (2014). Analogical Thinking Induces Eye Movements in Scene Viewing. International Journal of Machine Learning and Computing, 4(1), 94-98. http://dx.doi.org/10.7763/ijmlc.2014.v4.393

Ahmad, G. (2014). Eye Movements Look for Analogical Patterns during Scene Viewing. Journal of Economics, Business and Management, 2(4), 285-288.

Ahmad, G. (2013). Analogical Thoughts Persuade Eye Movements during Scene Viewing. International Journal of Intelligent Information Processing, 4(4), 39-45.

Ahmad, G. (2013). Analogy Influences Eye Movements during Scene Viewing. Proceedings of International Conference on Computing and Convergence Technology, Republic of Korea, (pp. 1-4).

Ahmad, G. (2011). Cognitive Impact of Eye Movements in Picture Viewing, International Journal of Intelligent Information Processing, 2(1), 1-8. http://dx.doi.org/10.4156/ijiip.vol2.issue1.1

Bird, G. D., Lauwereyns, J., \& Crawford, M. T. (2012). The role of eye movements in decision making and the prospect of exposure effects. Vision Research, 60, 16-21. http://dx.doi.org/10.1016/j.visres.2012.02.014

Bly, B. M., \& Rumelhart, D. E. (Eds.). (1999). Cognitive Science. USA: Academic Press.

Bojko, A. (2013). Eye Tracking, The User Experience: a practical guide to research. New York: Rosenfeld Media.

Brown, T. (1999). The Science and Art of Tracking. New York: NY: Berkley Books.

Duchowski, A. (2003). Eye Tracking Methodology: Theory and Practice. London: Springer Verlag.

Gentner, D. (1983). Structure-mapping: A theoretical framework for analogy. Cognitive Science, 7, 155-170. http://dx.doi.org/10.1207/s15516709cog0702_3

Gentner, D., \& Markman, A. B. (1997). Structure-mapping in analogy and similarity. American Psychologist, 52, 45-56. http://dx.doi.org/10.1037/0003-066X.52.1.45 
Gentner, D., \& Medina, J. (1998). Similarity and the development of rules. Cognition, 65, 263-297. http://dx.doi.org/10.1016/S0010-0277(98)00002-X

Gentner, D., Rattermann, M. J., \& Forbus, K. D. (1993). The roles of similarity in transfer: Separating retrievability from inferential soundness. Cognitive Psychology, 25, 524-575. http://dx.doi.org/10.1006/cogp.1993.1013

Gompel, R. P., Fischer, M. H., Murray, W. S., \& Hill, R. L. (Eds.). (2007). Eye Movements: A Window on Mind and Brain. UK: Elsevier.

Granka, L. A., Joachims, T., \& Gay, G. (2004). Eye-Tracking Analysis of User Behavior in WWW Search. SIGIR 04. Sheffield, South Yorkshire, UK: ACM. http://dx.doi.org/10.1145/1008992.1009079

Griffin, Z. M. (2004). Why look? Reasons for eye movements related to language production. In H. a. Ferreira (Ed.), The integration of language, vision, and action: Eye movements and the visual world (pp. 213-247). New York: Psychology Press.

Henderson, J. M., \& Hollingworth, A. (1998). Eye movements during Scene Viewing. An Overview. In G. W. Underwood (Ed.), Eye Guidance in Reading and Scene Perception (pp. 269-293). Oxford: Elsevier. http://dx.doi.org/10.1016/B978-008043361-5/50013-4

Henderson, J. M., \& Hollingworth, A. (1999). High-level Scene Perception. Annual Review of Psychology, 50(1), 243-271. http://dx.doi.org/10.1146/annurev.psych.50.1.243

Hermansen, O. (2015). Implementing eye movements in business applications. Brage Bibsys, Hogskolen i Ostfold.

Hoffman, J. E. (1998). Visual Attention and Eye Movements. In H. Pashler (Ed.), Attention (pp. 119-153). London: Psychology Press.

Hofstadter, D., \& Sander, E. (2013). Surfaces and Essences: Analogy as the Fuel and Fire of Thinking. New York: Basic Books.

Holsanova, J. (2008). Discourse, Vision, and Cognition. New York: John Benjamins Publishing Company.

Koller, M., Salzberger, T., Brenner, G., \& Walla, P. (2012). Broadening the range of applications of eye-tracking in business research. Analise, Porto Alegre, 23(1), 71-77.

Liversedge, S. P., Gilchrist, I. D., \& Everling, S. (2011). The Oxford Handbook of Eye Movements. Oxford University Press. http://dx.doi.org/10.1093/oxfordhb/9780199539789.001.0001

Nikolaev, A. R., Pannasch, S., Ito, U., \& Belopolsky, A. (Eds.). (2014). Eye movement-related brain activity during perceptual and cognitive processing. Frontiers Research Topics. Frontiers E-books. 
Orquin, J. L., \& Loose, S. M. (2013). Attention and choice: A review on eye movements in decision making. Acta Psychologica, 144(1), 190-206. http://dx.doi.org/10.1016/j.actpsy.2013.06.003

Pan, B., Hembrooke, H. A., Gay, G. K., Granka, L. A., Feusner, M. K., \& Newman, J. K. (2004). The Determinants of Web Page Viewing Behavior: An Eye-Tracking Study. Association for Computing Machinery, 147-154. http://dx.doi.org/10.1145/968363.968391

Pan, B., Hembrooke, H., Joachims, T., Lorigo, L., Gay, G., \& Granka, L. (2007). In Google We Trust: Users' Decisions on Rank, Position, and Relevance. Journal of Computer-Mediated Communication, $\quad 12, \quad 801-823$. http://dx.doi.org/10.1111/j.1083-6101.2007.00351.x

Rayner, K. (1992). Eye movements and visual cognition: scene perception and reading. New York: Springer Verlag. http://dx.doi.org/10.1007/978-1-4612-2852-3

Snowden, R., Thompson, P., \& Troscianko, T. (2012). Basic Vision: An introduction to visual perception. Oxford University Press.

Solso, R. L. (1994). Cognition and Visual Arts. London: MIT Press.

Solso, R. L. (2003). The psychology of art and the evolution of the conscious brain. MIT Press.

Stark, L., \& Ellis, S. R. (1981). Scanpaths Revisited: Cognitive Models Direct Active Looking. In R. A. Dennis F. Fisher (Ed.), Eye Movements: Cognition and Visual Perception (pp. 193-226). Hillsdale: Lawrence Erlbaum Associates.

Tommasi, L., Peterson, M. A., \& Nadel, L. (Eds.). (2009). Cognitive Biology: Evolutionary and Developmental Perspectives on Mind, Brain and Behavior. London: MIT Press. http://dx.doi.org/10.7551/mitpress/9780262012935.001.0001

Tsotsos, J. K. (2011). A Computational Perspective on Visual Attention. London: MIT Press. http://dx.doi.org/10.7551/mitpress/9780262015417.001.0001

Turner, M. (2006). The Artful Mind - Cognitive Science and the riddle of human creativity. Oxford University Press.

VanRullen, R., \& Thorpe, S. J. (2001). The time course of visual processing: from early perception to decision-making. Journal of Cognitive Neuroscience, 13(4), 454-461.

Viegas, F. B., \& Wattenberg, M. (2007). Artistic Data Virtualization: Beyond Visual Analytics. Second International Conference on Online Communities and Social Computing, (pp. 182-191).

Wells, A. (2000). Emotional Disorders and Metacognition: Innovative Cognitive Therapy. West Sussex: John Wiley \& Sons. http://dx.doi.org/10.1002/9780470713662 


\section{Copyright Disclaimer}

2016, Vol. 8, No. 2

Copyright for this article is retained by the author(s), with first publication rights granted to the journal.

This is an open-access article distributed under the terms and conditions of the Creative Commons Attribution license (http://creativecommons.org/licenses/by/3.0/). 